\section{Gründliche Reinigung von Medizininstrumenten}

Chirurgie, Orthopädie und Traumatologie sind typische Einsatzgebiete für die medizintechnischen Sterilisierungsbehälter und Instrumentenboxen der LK Mechanik. Dabei handelt es sich um einsatzfertige Komplettlösungen aus Edelstahlträgern und Silikonaufnahmen, die mit verschiedenen Verfahren wie dem Laserfeinschneiden, dem WasserstrahlFeinscheiden, dem Präzisionsstanzen und dem Laserschweißen genau an die Form der zu reinigenden Instrumente oder Implantate angepasst

\section{Handliches}

\section{Schichtdickenmessgerät für viele Einsatzgebiete}

Für die schnelle und genaue zerstöauf metallischen Untergründen eignet sich das handliche Schichtdickenmessgerät Layercheck 750 USB von Erichsen. Typische Einsatzbereiche sind der industrielle Korrosionsschutz, Galvanisierund Lackierbetriebe, die chemische Industrie sowie der Automobil- und Maschinenbau. Die Gummiumrandung bietet zusätzlichen Stoßschutz. Zum Lieferumfang gehört eine Sonde. Diese wird an einer federnden Griffhülse gehalten. Dadurch lässt sich die Sonde bei konstanter Auflagekraft sicher und kippfrei aufsetzen. Die einpolige Messsonde ist über ein Kabel (Länge $1 \mathrm{~m}$ ) mit dem Gerät verbunden. Für Messungen in dunkler Umgebung ist das batteriebetriebene Schichtdickenmessgerät mit einem großflächigen, beleuchteten LC-

Display ausgestattet. Die Schichtdickenmessgeräte arbeiten je nach Gerätetyp und Sonde nach dem magnetisch-induktiven Verfahren (Modell 750 USB-F) oder zusätzlich auch nach dem Wirbelstrom-Verfahren (Modell 750 USB-FN).

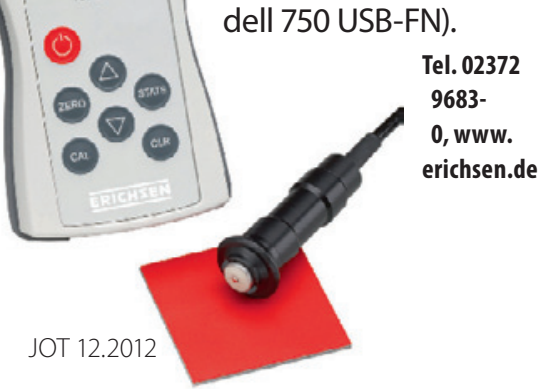

werden. Dies ist eine wesentliche Grundlage für eine gründliche Reinigung. Die Edelstahlträger wiederum eignen sich sowohl für die manuelle als auch automatisierte Reinigung. Auch sie werden genau auf die Gestalt der Instrumente abgestimmt. Bei den neuen Endoskop-Trägern wurden beispielsweise die Lochungen im Boden vergrößert, um den Wasserfluss zu beschleunigen, die Bildung von Spülschatten zu verringern und Trockenzeit zu reduzieren. Der Hersteller bietet auch Sonderanfertigen für ver-
$D$ e Firma Anest Iwata erweitert ihr Angebot an Doppelmembranpumpen mit zwei neuen Modellen; der DPS120 und der DDP70. Die Geräte eignen sich insbesondere für die Versorgung von Profi-Lackierpistolen wie zum Beispiel der W-200-Kesselhand- Lackierpistole oder der WAM-220-MangustaAutomatik-Lackierpistole. Die Pumpen verfügen über einen Doppelspül-Luftkreislauf und totraumarme Materialkanäle. Eine pneumatische Zwangsumsteuerung verhinder+ den Stillstand der Pumpe (Steuerung entspricht EN12621). Inspektion und Reinigung werden durch den zerlegbaren verschleißarmen Pumpenaufbau erleichtert. Die DPS120 arbeitet mit einer Förderleistung von $30 \mathrm{l} /$ min. Montiert auf einem fahrbaren Wagen ist dieses Modell entweder mit Ansaugrohr oder mit einem 6-I-Kunststofftrichter in den Ausführungen Edelstahl (15,5 kg) sowie Alumini- schiedene Reinigungs- und Sterilisierungsverfahren an.

Tel. 0641 96242-0, www.lk-mechanik.de

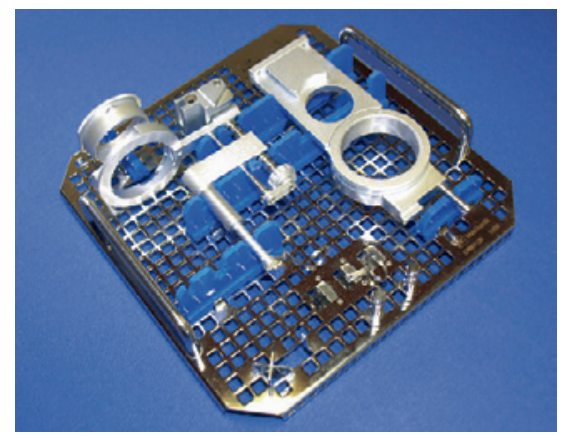

\title{
Doppelmembranpumpen zur Versorgung von Profi-Lackierpistolen
}

um eloxiert $(13,0 \mathrm{~kg})$ erhältlich. Die DDP70 hat eine Förderleistung von $5 \mathrm{l} / \mathrm{min}$. Sie ist in Edelstahl gefertigt $(3,2 \mathrm{~kg})$ und nicht vormontiert, lässt sich aber leicht auf einen Eimer des Herstellers montieren. Materialausgangs-, Lufteingangs- und Luftausgangsdruck betragen sowohl bei beiden Geräten 7,0 bar.

Tel. 0341 241443-0, www.anest-iwata.de 